\title{
A Business Collaborative Decision Making System for Network of SMEs
}

\author{
Muhammad Naeem ${ }^{1, *}$, Néjib Moalla ${ }^{1}$, Yacine Ouzrout ${ }^{1}$, Abdelaziz Bouras $^{2}$, \\ ${ }^{1}$ Decision and Information for Production Systems (DISP), \\ University Lumière Lyon 2, France \\ \{FirstName.LastName\}@univ-lyon2.fr \\ ${ }^{2}$ CSE Department, Qatar University Doha, Qatar \\ abdelaziz.bouras@qu.edu.qa
}

\begin{abstract}
The enterprise collaboration has gain much popularity and strength with the inception of digital revolution. The concept of enterprise collaboration has observed a dynamic and evolving phenomenon of value added chain. This phenomenon under the convergence of information technology has placed a remarkable impact on decision-making processes within enterprises. The enterprises are involved in establishing a common window of collaborative network where the principle enterprise decides the synthesis of the incoming opportunity. In this study, we have shown how the decision-making capability can be improved by means of analytics of vast amount of data during enterprise collaboration. The proposed system has adopted prescriptive analysis across enterprise resources. The outcome of proposed system addresses the individual and collaborative enterprise capability within enterprise network. Our proposed knowledge based decision making model provides a self-adaptive solution for enterprise collaborative services.
\end{abstract}

Keywords: big data, enterprise collaboration, opportunity analysis, capability evaluation, data asset

\section{Introduction}

Since the inception of shift of paradigm of manufacturing from 'whole' product to 'integrated' product, the Small and Medium Enterprises (SMEs) are facing operational challenges in turbulent environment [1]. This phenomenon is directing the SMEs towards improving the quality of decision-making process. The dynamic and in time momentum of quality aware decision making process has shaped itself into a part and parcel of the enterprise management. The information support for decision-making processes at all levels of the enterprise is inevitable. Moreover, the organizational arrangement of the processes are becoming increasingly important. The processes in the enterprises are meant to produce data all the time. The accumulation of data across the business processes leads to the technical challenge of handling Big Data (BD) in enterprises in a value-driven course of action. Big Data amounts not only towards 
data gathering but, most of all, data processing, organization and its visualization. All of these are in fact substantial for obtaining business improvements.

Data warehouses are a conventional means of storing and processing BD. Data warehouse environment is traditionally a production driven, equipped with highly managed service level agreements [2]. This environment certifies timely generation of managerial reports as well as dashboards. Certainly, it is a cost effective job where a skilled person is required to organize newly generated data source. Such an idea is marked by the persistent granular data along its traceable history in the data warehouse system. This conventional approach plays in an environment of highly structured data warehouse [2]. We have proposed an added value to this traditional approach in business analysis coined by our earlier big data framework [1], [3].

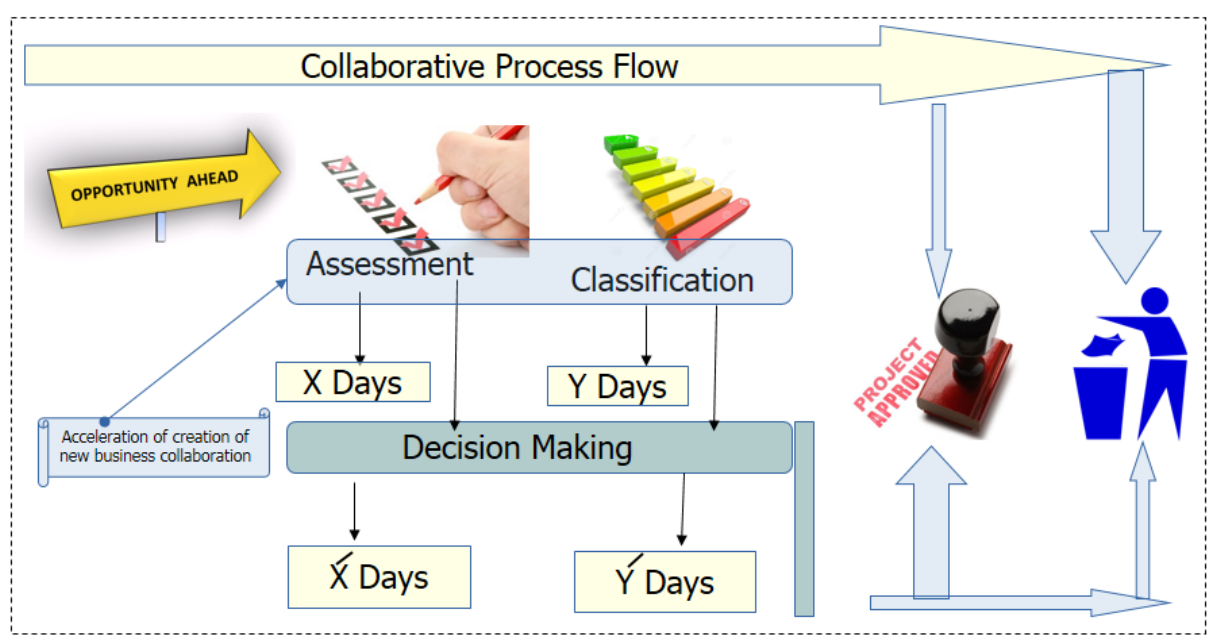

Fig. 1. A motivational scenario of early response by means of enterprise collaborative decision making.

We present a simple case scenario (see the Fig. 1) for the requirement of collaborative network. An enterprise is receiving large number of projects. There is a likelihood of any project to be an opportunity. We already described that the competitive environment is pushing enterprises to leverage more and more attractable versatility in the products. Under normal circumstances, numerous projects are deemed to be inappropriate because the enterprise has no mechanism to extrapolate the level or degree of collaborative effort required in transforming the 'risky' projects into acceptable projects. The decision making system can first reduce the timing for initial assessment and classification of the acceptance of the projects; henceforth the rate of the profitable projects is significantly increased. The motivation is to establish an in house decision making facility of enterprise collaboration where the data anonymity is also maintained. The reason for establishing the enterprise collaboration coined by data anonymity is marked by the fact that the enterprises are always prone to be sensitive in exposing their data pertaining to its internal business processes.

Our approach is to generate a step forward analytic sandbox with the slogan of loading whatever data is available from internal and external data sources. The idea is 
to perform more data profiling, refining, transformation, identifying and generating new composite metrics. Keeping in view of this added value, the proposed system takes the structured data as well as Computer Aided Design (CAD) design resource file STandard for the Exchange of Product model data (STEP) [4] file and integrate both of them for the purpose of a "Forehand Analysis". Here the term "Forehand Analysis" refers to the situation in which firstly an enterprise is receiving sufficient number of projects. Secondly, each project may or may not be a profitable opportunity or waste of time depending on its context. Thirdly, the enterprise needs to produce a positive or negative response as soon as possible.

The remaining paper is organized into three sections. The section 2 is related to conceptual and pragmatic work related to the theme of this study. In section 3, we have shown our methodology. The section four is dedicated to explanation of a holistic approach using all components in section 3. The paper in last section is concluded with some recommendations and future extensions.

\section{Related Work}

We have drawn a specialized taxonomy of application of BD in decision making of enterprise collaboration. The applications of BD analytics can be classified into three broader categories including descriptive, predictive and prescriptive analytics.

The term "descriptive analytics" briefly refers to "What has been done". The descriptive analytics is carried out in two situations. The first situation is a frequency based approach usually obtained by means of aggregation across source data. The second is on demand as in case of on-line analytical processing. The descriptive analysis is characterized by the identification of problems by means of drilling down into the ongoing processes, their technical and functional detail within the organization. We have noticed that most the work in this category is performed in social alliance [5], strategies in financial alliance [6], internationalization [7], supply chain [8], and unexpected events management in the context of hierarchical production planning [9] and others [10].

The predictive analytics is aimed towards identifying what is happing in future or what type of new instance can be generated. It is also coined by inference mechanism in data analytics. Apart from this, it can also be used for granular level grouping of the existing instances in shape of a complex tree or graph [11]. It can be used for the inference and forecasting purpose as well $[12,13,14]$. The Table 1 is showing the detail for the collaborative work performed in the area of communication enabling and logistic industry.

Some researchers have also defined another type of analytics known as prescriptive analysis $[15,16,17]$. Its scope is broader but at the same time it draws the cumulative effect of complexity. It is aimed towards the exploitation of data as well as the algorithms to find the most appropriate (or nearest) decisions targeting objectives and requirement related to high volume of data and its versatile nature. It can be briefly described as "What is suggested to be done". It is know that BD has already provided an umbrella of NoSQL where complex structures or semi structured can be handled by means of storage into key value, column family database, document family and 
graphical database. The Industries are producing more and more versatile nature of data. This aspect accentuates the needs of leveraging the NoSQL technologies for descriptive and predictive learning. Both of these learning analytics play a vital role in helping companies realizing effective decisions in the strategic direction of the organization. We argue that a more holistic approach by means of utilizing these two fundamental learning system accompanied by knowledge base learning can realize into a best course of learning action namely prescriptive analysis. It qualifies the problem of enterprise collaboration a good candidate under the prescriptive analysis. But on the other hand, one can conclude from the literature review that operational level enterprise collaboration in perspective analysis is still missing.

Table 1. Big Data Analytic in enterprise collaboration

\begin{tabular}{llll}
\hline & \multicolumn{1}{c}{ Descriptive } & \multicolumn{1}{c}{ Predictive } & \multicolumn{1}{c}{ Prescriptive } \\
\hline Enterprise & - supply chain [8] & - forest enterprises & - collaboration \\
Collaboration & - social alliance [5] & [11] & \\
Strategy & - financial alliance [6] & & \\
& & & \\
Enterprise & - work flow, mappings & - communication & - safety \\
Collaboration & between activities in & enabling [12] & management [15] \\
Planning & $\begin{array}{l}\text { process models [10] } \\
\text { - Internationalization [7] }\end{array}$ & & \\
& & & \\
Enterprise & - unexpected events & - Logistic industry & - \\
Collaboration & management in the context & [14] & \\
Operation & $\begin{array}{l}\text { of hierarchical production } \\
\text { planning [9] }\end{array}$ & $\begin{array}{l}\text { - Content } \\
\text { Management [13] }\end{array}$ & \\
\hline
\end{tabular}

To extend the concept of perspective level data analytics in enterprise collaboration, we introduced the non-conventional resources other than relational data. The CAD model can be considered for the purpose of identification of explicit or implicit manufacturing capabilities. Since the inception of the concept of PLM and the emergence of the STEP CAD format [4], the research community has been motivated to introduce the mechanism of extracting the low level geometric entities of a product design. The STEP format holds a specific structure which is typically used to facilitate process planning and manufacturing activities [4].

\section{Proposed Methodology}

We previously pointed out gaps in the coherent implementation of the data analytics in the pursuit of enterprise collaboration. Our methodology is based on the conceptual design to bring out a prescriptive analysis suitable for the decision making. We shall propose the generic steps to obtain the idea formulated on the basis of the gap identified in the literature review. The Fig. 2 is showing the steps for the treatment and analysis of the incoming project from the stream of opportunities. The STEP [4] file is analyzed into two parts / section. One section (plastic) is related to the 
manufacturing domain of enterprise and the second component is the realm of metal production. The metal production facility is the business of another organization which is a potential partner enterprise as well. To complete this project, both of the enterprises are in need of a profit oriented collaboration. Our system will analyze the design structure (available from STEP resources).

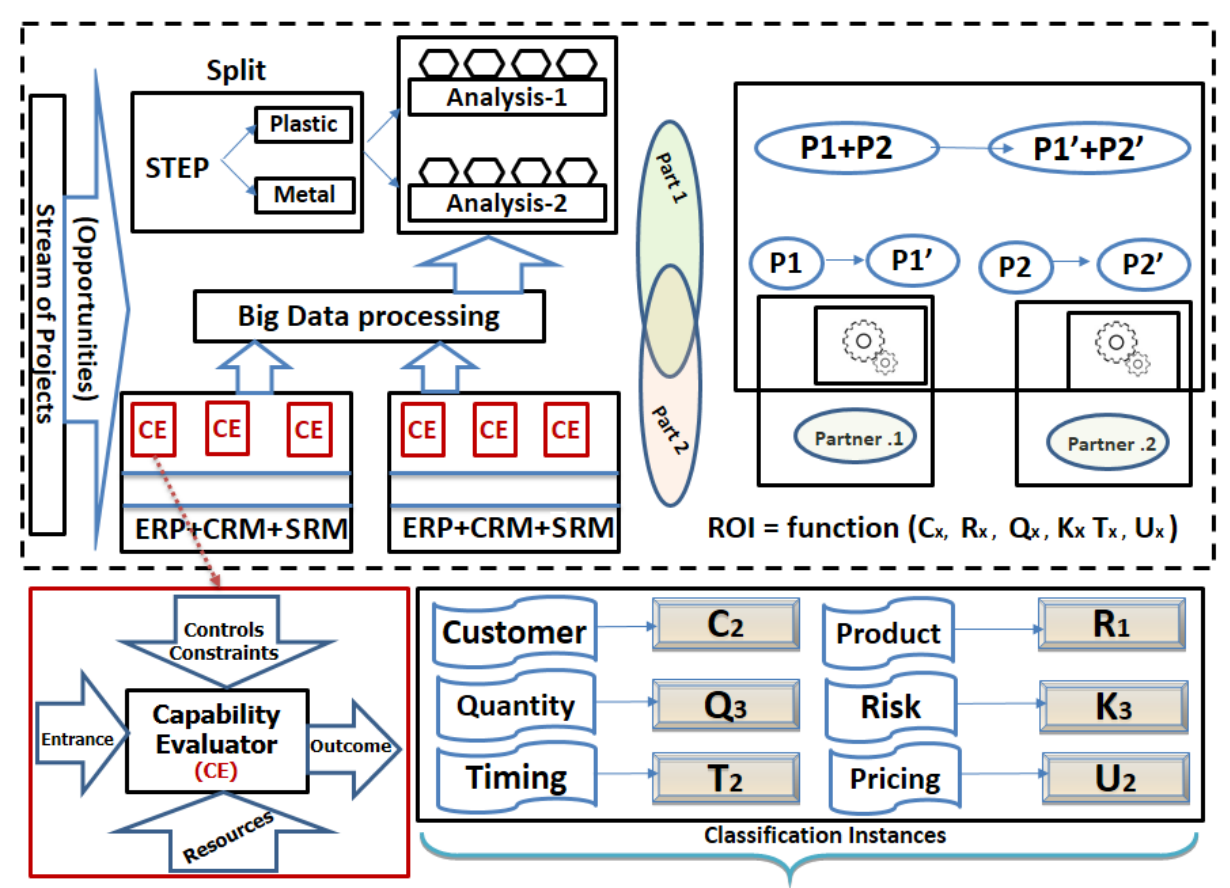

Fig. 2. Methodology of classification of technical and functional characteristics during decision making treatment for enterprise collaboration.

However, synthesis of STEP resource is insufficient till it is backed up by other data, specially the transformation performed by BD analytics. We in our system adopt this data from the Supplier Relationship Management (SRM), Product Life cycle Management (PLM), Customer Resource Management (CRM) and Enterprise Resource Planning (ERP) in an iterative way. From a historical perspective of information system application, ERP is comprised of several integrated modules. These include logistics, procurements, sales, marketing, human resources and finance supporting intra organizational collaboration. SRM extends its scope to include market information with focus upon order management for improving product availability and enhancing customer satisfaction. All of these structured data provides the array of transactions related to customer, product, costing, taxation and timing records related to the previous similar projects. A combine analysis of both of these stream of resources is likely to produce the classification as shown in the classification instances in the Fig. 2. 
Table 2. Capability Evaluator - parameters for product wise ranked list of partner

\begin{tabular}{llll}
\hline $\begin{array}{l}\text { Inputs } \\
\text { (Entrance) }\end{array}$ & $\begin{array}{c}\text { Controls } \\
\text { Constraints }\end{array}$ & Outcome & Resources \\
\hline - List of partner & - Last x months & - Ranked list of partner (general) & - Partners \\
- Specific & of business with & - Ranked list of partner (for a & - Orders \\
opportunity & each partner & specific opportunity / project) & - Quotation \\
\hline
\end{tabular}

The Capability Evaluator (CE) is the technical transformation component. This component is based on statistical descriptive or predictive analysis. Each $\mathrm{CE}$ outcomes a classification illustrating the capability of each enterprise individually. It is useful that we explain the components responsible for the analysis of structured data. The generic functionality and specification of every component is characterized by consumption of input resources. It operates on the input by means of given resources under certain constraints and controls. Each component produces an output which functions the classification system of the enterprise capacity in specific context of the system. We shall discuss a few CE components with its functional capability and technical specification as shown in Tables 2 and 3.

Ranking the partner is an important treatment in establishing the enterprise collaborative facility. To illustrate the functional detail of product wise ranked list of partners. Let us assume that there is a collection of orders placed by the potential customers. Each of the orders itself contains a detail of the product. The input for the ranking is not limited to merely the placement of the fresh orders. It also incorporates the previous list of the orders which were either regretted, successfully completed or just finished with trivial accomplishments.

Table 3. Capability Evaluator - parameters for risk likelihood (outcome: Causation risk model)

\begin{tabular}{lll}
\hline $\begin{array}{l}\text { Inputs } \\
\text { (Entrance) }\end{array}$ & \multicolumn{1}{c}{ Controls Constraints } & Resources \\
\hline $\begin{array}{l}\text { History of } \\
\text { specific }\end{array}$ & - change in the price of the raw material & - Sentiment analysis \\
- product item & - new taxation and levies (if any) & - Project detail \\
- customer & - intrinsic level of the design of the product & - Product design \\
- potential & - profit margin & \\
partner & - insurance liability/coverage & \\
\hline
\end{tabular}

In ranking, given a query, the ranking function assigns a score to each partner, and ranks the partner in descending order of the scores. The ranking order represents the relevance of partner with respect to the query. In learning the model, a number of queries are provided; each query is associated with a perfect ranking list of partner; a ranking function is then created using the training data. This process is carried out in a way that the model can precisely predict the ranking lists in the training data. The problem is addressed by means of pair-wise approach as carried out by Cao et al. [18] in their document retrieval problem or by means of a ranking modeling.

Risk is an inevitable characteristic associated with every incoming opportunity. It varies widely from customer to customer, opportunity to opportunity. It demands an in depth understanding of other related factors. An in depth understanding of overall 
risk assessment is likely to help predict the likelihood and cost of incoming opportunity. Numerous conditions contribute to the frequency and severity of the associated risks. These include ignoring the drastic change in the price of the raw material, new taxation and levies (if any), environmentally affected production process, intrinsic level of the design of the product, profit margin, insurance liability/coverage etc. The goal of this module is to provide a realistic assessment based on the characteristics of the incoming opportunity in the shape of a project.

\section{$4 \quad$ Holistic Enterprise Collaboration}

In previous section, we provided two higher level of CE in Table 2 and 3. We have termed the descriptive level of CE in Level 1 (CE.L1) and those which require a higher level of course of action are placed under the category of CE in Level 2 (CE.L2). In our research the final objective of decision making for an incoming opportunity (project) is not limited to only two CE.L2. In-fact, there are numerous such CE.

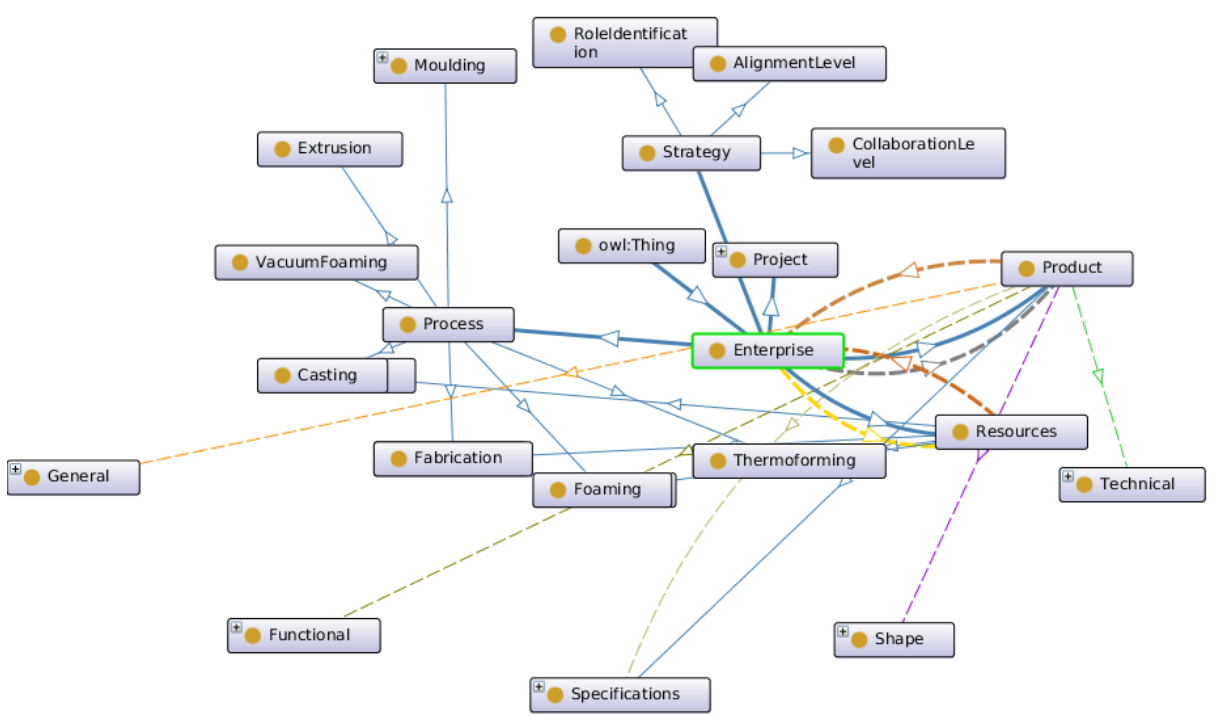

Fig. 3. A top level concept view of enterprise collaboration value ontology.

The collective and accumulative effect of all of these CE irrespective of their level plays a non-trivial role in determination of a prescriptive decision making system. The outcome of these $C E$ are shaped in either the classification such as $C_{x}, Q_{x}, R_{x}, T_{x}$ etc., (see the Fig. 1 for their interaction and outcome). All of these CE return an assessment in the shape of Return of Investment $(R o I)$ as can be defined:

$$
R o I=f\left(C E_{I}, C E_{2}\right)
$$


The RoI (defined in equation 1) is certainly an essential transformation for the decision making. Here question arises how this prescriptive analysis in form of RoI can be achieved. The answer is provided by means of ontological modelling. The Table 4 is showing the mapping, interaction and evolution of knowledge base. The interaction is focused on ontological engineering of the CE interoperability and domain knowledge representation (shown in Fig. 3). A repository of semantically encoded rules using SWRL queries for dynamic decision making will bring forward the decision about the suitability of an incoming opportunity. The whole decision making system is not only dynamic but also iterative. The continuous evolution and refinement of core value ontology gives a clear advantage of those system which either use conventional database system or connect the knowledge base directly to the underlying first level (raw) data.

Table 4. A partial overview of mapping of knowledge base (BD analytics) to ontology schema.

\begin{tabular}{ll}
\hline Property / Relationship across T-Box & Knowledge Base from CE \\
\hline In-house / partner Mechanical Processes & Aggregated numeric value (L1) \\
In-house / partner cost & Frequency based value (L1) \\
In-house / partner Timing & Time series analysis (L2) \\
Partner Recommendation Index & Random forest prediction (L2) \\
x months business volume with partner & Frequency based value (L1) \\
Change in the price of the raw material & Frequency based value (L1) \\
Taxation / Levies & Frequency based value (L1) \\
Risk Factor & Naive Bayes prediction (L2) \\
Customer Classification & Frequency based value (L1) \\
Collaboration Level & Regression analysis (L2) \\
Churn Index & Random forest prediction (L2) \\
\hline
\end{tabular}

\section{Conclusion}

The idea of enterprise collaboration is gaining a strategic position. This position is triggered by the ineluctable need of the enterprises to revitalize their chain of manufacturing with optimized utilization of underlying resources. Modern infrastructure of information technology is characterized by complex set of hardware, software along with its underlying enterprise policies and solutions processed with 
organizational resource. This complexity exercises an influence on the decision making of enterprise management and its collaborative effort. Given the contemporary collaborative operations, it is not a straight forward process to establish a strong connection between functional exploitation of versatile large amount of data and quality of decisions. To cope up this challenge, we have proposed a system treating various transformation and translation of $\mathrm{BD}$ capabilities into a dynamic decision making system. The outcome of this study is a decision making system across demands of versatile products. We have shown how the system can enhance support of the networked enterprise in timely creation and participation in collaborative environment by means of facilitating an infrastructure. The infrastructure is aimed towards discovery, capturing, delivery and application of this knowledge to create collaboration in improvement of operational efficiency. A possible extension to this work lies in the preservation of the decision making rules in such a shape which can facilitate the forthcoming opportunities and projects.

\section{References}

1. Naeem, M., Moalla, N., Bouaras, A., Ouzrout, Y.: Opportunity Analysis for Enterprise Collaboration between Network of SMEs. (2015).

2. Kościelniak, H., Puto, A.: BIG DATA in Decision Making Processes of Enterprises. 65, 1052-1058 (2015).

3. Naeem, M., Moalla, N., Bouaras, A., Ouzrout, Y.: Weaving Trending, Costing and Recommendations Using Big Data Analytic: An Enterprise Capability Evaluator. In: Enterprise Interoperability in the Digitized and Networked Factory of the Future, Proceedings of the I-ESA Conferences, Guimares, Portugal (2016).

4. Iso, I.S.: 10303-1 TC184/SC4: Product Data Representation and Exchange-Part 11: The EXPRESS Language Reference Manual. (1994).

5. Sakarya, S., Bodur, M., Yildirim-Öktem, Ö., Selekler-Göksen, N.: Social alliances: Business and social enterprise collaboration for social transformation. $65,1710-1720$ (2012).

6. Kaya, N.: Corporate Entrepreneurship, Generic Competitive Strategies, and Firm Performance in Small and Medium-sized Enterprises. 207, 662- 668 (2015).

7. Costa, E., Soares, A.L., Sousa, J.P. de: Information, knowledge and collaboration management in the internationalisation of SMEs: A systematic literature review. $36,557-569$ (2016).

8. Hoof, B. van, Thiell, M.: Collaboration capacity for sustainable supply chain management: small and medium-sized enterprises in Mexico. 67, 239- 248 (2014).

9. Vargas, A., Boza, A., Patel, S., Patel, D., Cuenca, L., Ortiz, A.: Inter-enterprise architecture as a tool to empower decision-making in hierarchical collaborative production planning. - (2015).

10. Tan, W., Li, L., Xu, W., Yang, F., Jiang, C., Yang, L., Choi, J.: A role-oriented service system architecture for enterprise process collaboration. 39, 1893- 1900 (2012). 
11. Ambrose-Oji, B., Lawrence, A., Stewart, A.: Community based forest enterprises in Britain: Two organising typologies. 58, 65- 74 (2015).

12. Kolberg, M., Buford, J.F., Dhara, K., Wu, X., Krishnaswamy, V.: Feature interaction in a federated communications-enabled collaboration platform. 57, 2410- 2428 (2013).

13. Nof, S.Y., Morel, G., Monostori, L., Molina, A., Filip, F.: From plant and logistics control to multi-enterprise collaboration. 30, 55- 68 (2006).

14. Pateman, H., Cahoon, S., Chen, S.-L.: The Role and Value of Collaboration in the Logistics Industry: An Empirical Study in Australia. 32, 33- 40 (2016).

15. Niskanen, T., Louhelainen, K., Hirvonen, M.L.: Results of the Finnish national survey investigating safety management, collaboration and work environment in the chemical industry. 70, 233- 245 (2014).

16. To, C.K.M., Ko, K.K.B.: Problematizing the collaboration process in a knowledge-development context. 69, 1604- 1609 (2016).

17. Wang, G., Gunasekaran, A., Ngai, E.W.T., Papadopoulos, T.: Big data analytics in logistics and supply chain management: Certain investigations for research and applications. 176, 98-110 (2016).

18. Cao, Z., Qin, T., Liu, T.-Y., Tsai, M.-F., Li, H.: Learning to rank: from pairwise approach to listwise approach. In: Proceedings of the 24th international conference on Machine learning. pp. 129-136. ACM (2007). 